IMECE2004-61918

\title{
HYDRODYNAMICS OF CEREBROSPINAL FLUID IN SPINAL CANAL WITH CHIARI MALFORMATION AND SYRINGOMYELIA
}

\author{
Wojciech Kalata \\ University of Illinois at Chicago \\ Department of Mechanical and Industrial Engineering \\ Chicago, Illinois \\ John Oshinski \\ Emory University \\ Department of Radiology \\ Atlanta, Georgia
}

\author{
Bryn Martin \\ University of Illinois at Chicago \\ Department of Mechanical and Industrial Engineering \\ Chicago, Illinois \\ Francis Loth \\ University of Illinois at Chicago \\ Department of Mechanical and Industrial Engineering \\ Chicago, Illinois
}

\begin{abstract}
Magnetic resonance imaging (MRI) is has great potential as a tool for diagnosis of neurological diseases such as Chiari malformation (CM) and syringomyelia (SM). Its extended capability to obtain in-vivo velocities of blood or cerebrospinal fluid (CSF) allowed engineers to perform studies with engineering analysis applications such as computational fluid dynamics and physical modeling. Recently, a new MR technique called balanced steady-state free procession (bSSFP) cine imaging was developed for analysis of geometrical changes during the cardiac cycle due to compliance [1].

This study used MRI utilities to investigate hydrodynamic environment of CSF in the sub-cranial subarachnoid space (SAS). A model was constructed to simulate fluid dynamics of CSF in SAS. This model will allow investigation of detailed pressure and velocity using laser Doppler anemometry and pressure transducers. The MRI data from patient, healthy volunteers, the flow model were compared.
\end{abstract}

\section{INTRODUCTION}

CSF fluid moves in a pulsatile manner within the SAS of the spinal canal and cranial cavity. This fluid cushions the brain from impact on the cranial bone due to sudden motions. CSF also delivers nutrients and protein to and from the central nervous system and removes waste products.

$\mathrm{CM}$ is a disorder that consists of a downward displacement of the lower section of the brain (cerebellum tonsils) into the spinal canal. This disorder disrupts the normal hydrodynamics of CSF flow in the head and spinal canal. SM, which is a syrinx inside the spinal cord filled with CSF, can also develop in patients with CM or spine trauma.

This study is directed toward a better understanding of the hydrodynamic environment within SAS with CM and SM. Since geometry and flow rate can be obtained from the MRI, the pressure and velocity can be computed, as demonstrated by Kalata [2] and Loth et al. [3]. The characterization of the hydrodynamic environment using modeling may lead to greater understanding of pathogenesis of craniospinal disorders such as $\mathrm{CM}$ and SM.

\section{METHODS}

One CM/SM patient was scanned before decompression surgery (craniectomy). MRI geometries of the bottom half of the brain, upper spinal cord, and sub-cranial SAS were obtained. During the same scan session, cardiac gated phase encoded velocity was measured at four different axial positions of the upper back and neck (C2, C4, C7, and T3). Using inhouse developed software in Matlab, the flow waveforms were calculated at these locations.

The MRI anatomy images from the patient were used to reconstruct a rigid 3D Sylgard model of the SAS. The geometry of the blockage was complicated in shape particularly in the region where the brain tonsils descended and created narrow passages for CSF flow.

The 3D model of the sub-cranial SAS was reconstructed using commercial software MIMICS (Materialise, Ann Arbor, MI). Smoothing and extension additions were performed to insure the hydrodynamic reproducibility to the in-vivo condition. The spatial negative of the flow model was built with rapid prototyping machine using WaterWorks material (StrataySys, Eden Praire, MN). The SAS geometry was cast around the negative using Sylgard 184 (Dow Corning, Midland, MI). After curing, the negative was dissolved with a mixture of water and solvent using a temperature controlled ultrasonic tank.

The flow in the Sylgard model shown in Figure 1 was driven by pulsatile pump constructed from computer-controlled linear motor. Velocity measurements inside the model were 
performed using phase contrast MRI. At the location representing the in-vivo $\mathrm{C} 2$ level, measurements of all velocity components were obtained.

Additionally, three healthy volunteers and two CM patients were scanned with MRI for anatomy, velocity, and geometric changes due to compliance. The cross-sectional area of each spinal canal was investigated just below the base of the skull (C2 level). The cross-sectional area changes of the SAS were determined by carefully selecting the cut-off threshold pixel intensity value based on the largest image intensity gradient for each MRI slice. Then, addition of all the pixels that had higher intensity than the threshold value was performed to obtain the total cross-sectional area per image. For each case, there were $\sim 30$ images per cardiac cycle.

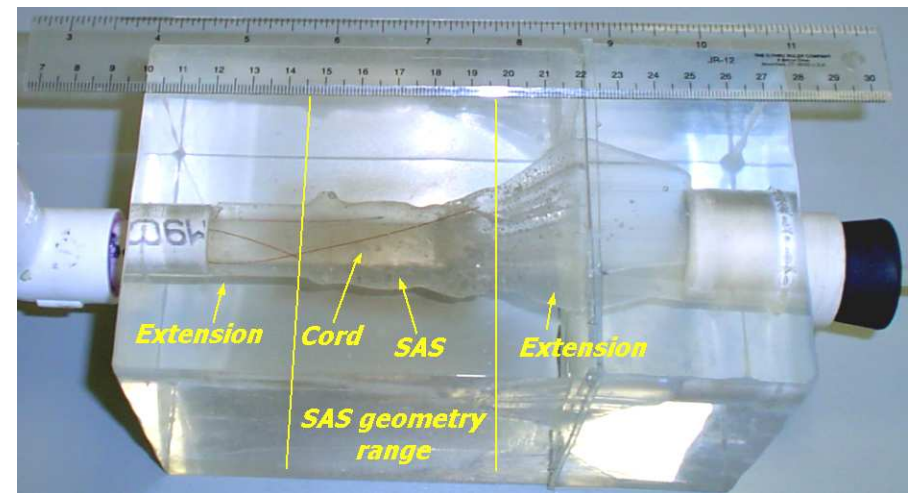

Figure 1. Sylgard model based on in-vivo geometry of SAS with CM. Head side is on the right.

\section{RESULTS/DISCUSSION}

The waveforms of the patient demonstrated similar magnitudes with a small phase shift of $0.05 \mathrm{~s}$ from the top (C2) to bottom (T3). The phase shifts occurred due to the compliant nature of the spinal cord and the thin layer of tissue along the spine. Flow inside the syrinx was detected at positions C7 and T3. The CSF peak systolic flows in the SAS were approximately 10 times higher than those in the syrinx.

The $\mathrm{C} 2$ waveforms of the patient and the model had similar magnitudes (Figure 2). Although their general trend was similar, the shapes of the flow waveforms were not identical especially with respect to acceleration. Figure 3 indicates that the velocities in the model show similar magnitudes as compared to the in-vivo velocities. In the in-vivo MRI flow images the spinal nerve roots were detected but in the model, the nerve roots were omitted for simplicity. The flow in the model was more uniform than in the in-vivo measurement. Pixel resolution of the phase contrast MR images of flow model was nearly two times greater than the patient's. Within the model at C2 level, the axial flow was dominant with small secondary flow (maximum $10-20 \%$ of axial flow).

Compliance of the spinal canal must exist due to the soft tissue of spinal cord as evidenced by the phase shift of flow waveforms at different axial locations. The change in SAS area was sometimes too small to be detected by MRI.

\section{CONCLUSIONS}

This study is directed toward better understanding of the hydrodynamics within the spinal canal and syrinx. This study demonstrated that the physical model reproduced in-vivo hydrodynamic environment in the CM/SM patient. Some cases had no significant area changes during the cardiac cycle, when analyzed with bSSFP cine MRI technique. The characterization of the hydrodynamic environment using modeling may lead to greater understanding of pathogenesis of craniospinal disorders such as CM and SM.

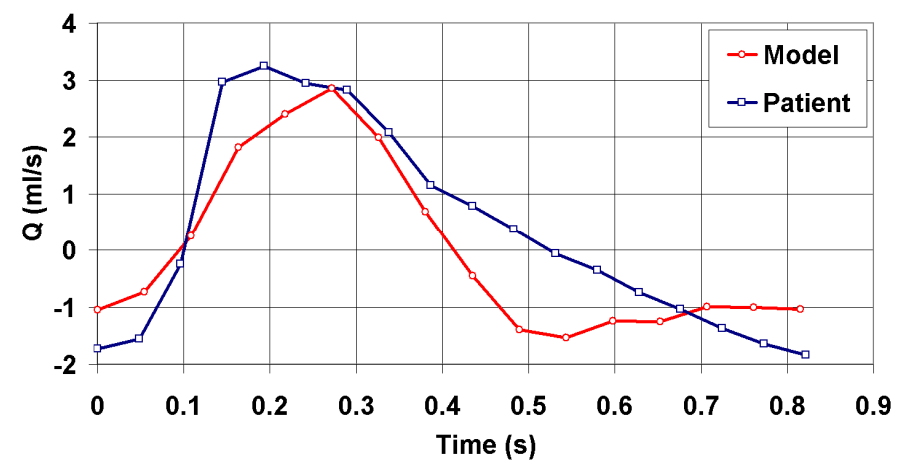

Figure 2. Flow waveforms from the CM/SM patient and model at $\mathrm{C} 2$ level with heartbeat at $69 \mathrm{bpm}$.
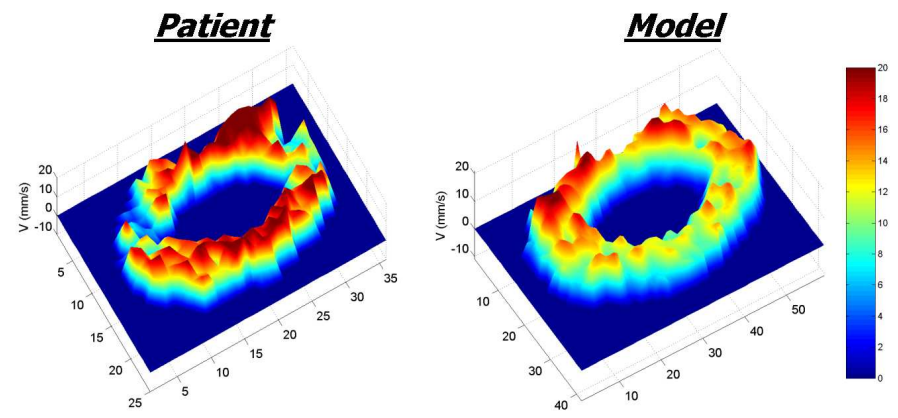

Figure 3. Velocity distribution for patient and model at $\mathrm{C} 2$ level during the systole peak (same scale for both plots ranging from 0 to $20 \mathrm{~mm} / \mathrm{s}$ ).

\section{ACKNOWLEDGMENTS}

This work was supported by American Syringomyelia Alliance Project. Wojciech Kalata would like to thank American Council for Polish Culture for a Pulaski Scholarship for Advanced Studies.

\section{REFERENCES}

1 Jin S., Oshinski J., Giddens D.P., 2003, "Effects of Inflow Conditions, Wall Motion and Compliance on Flow in the Ascending Aorta" Journal of Biomechanical Engineering, 125(3), pp. 347-54.

2 Kalata W., 2002 "Numerical simulation of cerebrospinal fluid motion within the spinal canal" Master thesis, Chicago, University of Illinois at Chicago.

3 Loth F., Yardimci M.A., Alperin N., 2001, "Hydrodynamic Modeling of Cerebrospinal Fluid Motion Within the Spinal Cavity", Journal of Biomechanical Engineering, 123, pp. 71-79. 